\title{
Retrosigmoid intradural suprameatal approach to Meckel's cave and the middle fossa: surgical technique and outcome
}

\section{Madjid Samii, M.D., Marcos Tatagiba, M.D., and Gustavo A. Carvalho, M.D. \\ Department of Neurosurgery, Hannover Medical School and Nordstadt Hospital, Hannover, Germany}

Object. The goal of this study was to determine whether some petroclival tumors can be safely and efficiently treated using a modified retrosigmoid petrosal approach that is called the retrosigmoid intradural suprameatal approach (RISA).

Methods. The RISA was introduced in 1983, and since that time 12 patients harboring petroclival meningiomas have been treated using this technique. The RISA includes a retrosigmoid craniotomy and drilling of the suprameatus petrous bone, which is located above and anterior to the internal auditory meatus, thus providing access to Meckel's cave and the middle fossa.

Radical tumor resection (Simpson Grade I or II) was achieved in nine (75\%) of the 12 patients. Two patients underwent subtotal resection (Simpson Grade III), and one patient underwent complete resection of tumor at the posterior fossa with subtotal resection at the middle fossa. There were no deaths or severe complications in this series; all patients did well postoperatively, being independent at the time of their last follow-up examinations (mean 5.6 years). Neurological deficits included facial paresis in one patient and worsening of hearing in two patients.

Conclusions. The approach described here is a useful modification of the retrosigmoid approach, which allows resection of large petroclival tumors without the need for supratentorial craniotomies. Although technically meticulous, this approach is not time consuming; it is safe and can produce good results. This is the first report on the use of this approach for petroclival meningiomas.

\section{Key Words * meningioma * Meckel's cave * petrous bone * surgical approach}

Abbreviations used in this paper: $\mathrm{CPA}=$ cerebellopontine angle; $\mathrm{CSF}=$ cerebrospinal fluid;

$\mathrm{CT}=$ computerized tomography; IAC $=$ internal auditory canal; IAM = internal auditory meatus; ICA = internal carotid artery; $\mathrm{MR}=$ magnetic resonance; $\mathrm{RISA}=$ retrosigmoid intradural suprameatal approach.

Different tumors may be encountered in the petroclival region, the most common being meningiomas affecting Meckel's cave and schwannomas of the trigeminal nerve. $[15,16]$ Other lesions include epidermoid cysts, chondrosarcomas, and chordomas.[18,20,22,25] Meningiomas and schwannomas are by far the most frequently occurring petroclival tumors. They usually extend into both the middle and posterior fossae and pass through Meckel's cave, which interconnects the fossae. These tumors may present with a major extension into the middle or posterior fossa, or they may have a dumbbell shape 
with equal extension into both fossae. The choice of surgical approach to deal with the lesion will depend largely on the type of tumor extension.

In the last two decades, major efforts have been made to improve surgical results in treating tumors that involve the petroclival area and extend into the posterior and middle fossae. In the past, several of these large tumors were considered inoperable; however, in the last 15 to 20 years more satisfactory results have been witnessed.[2-4,8,11,14,17,22,25] Advanced skull base techniques with extended exposures of the temporal bone have contributed enormously to improving tumor resectability and patient outcome. All these approaches (extended middle fossa, Kawase's approach, combined subtemporal--presigmoid exposure, and so forth) compose varying degrees of petrosectomy and tentorium division.[6,18,21,27]

With increased experience in dealing with these lesions, however, we have learned that a number of these tumors can be safely treated without major exposure of petrous bone structures.[19] Avoidance of large petrous bone resections may significantly reduce operative risks such as hearing loss, facial palsy, and CSF leak. In 1983, a modification of the classic retrosigmoid approach was introduced by the senior author (M.S.) in our department to treat some petroclival lesions.[18] This approach includes a retrosigmoid craniotomy and intradural drilling of the bone located above and anterior to the IAM. The drilling procedure involves the suprameatal area and the posterior portion of the petrous apex, which is located below and lateral to the trigeminal cavity. The latter allows for wider opening of Meckel's cave, with increased exposure and mobilization of the trigeminal nerve and access to the middle fossa. This exposure may be combined with division of the tentorium, which increases the corridor to the middle fossa. Therefore, we have called this approach the "retrosigmoid intradural suprameatal approach" or "RISA."

In 1995 Cheung, et al.,[5] reported in the otological literature a similar approach to resect a trigeminal schwannoma that was growing into the middle and posterior fossae. Dumbbell-shaped trigeminal schwannomas usually expand the bone surrounding Meckel's cave, creating a natural enlarged space between the posterior and middle fossae. This is the space that is gained via the RISA by drilling away the suprameatal bone dorsocaudal and lateral to Meckel's cave. Recently, Seoane and Rhoton[23] described in detail the microsurgical anatomy of the suprameatal approach, pointing out important neurovascular structures and landmarks related to this approach.

To study the impact of the RISA on surgical results in patients treated for petroclival tumors, we reviewed 12 cases of petroclival meningiomas that were resected using this approach in our department during the last 15 years. We have not included cases of trigeminal schwannomas, chondrosarcomas, or other petroclival lesions in this series.

\section{CLINICAL MATERIAL AND METHODS}

Between 1978 and 1997, 95 patients harboring meningiomas of the petroclival region were treated at the Department of Neurosurgery of Nordstadt Hospital in Hannover, Germany. A restrospective study of the medical records of these 95 patients showed that the tumors were resected using different approaches, including frontotemporal, subtemporal, retrosigmoid, combined retrosigmoid--subtemporal, and combined presigmoid--subtemporal approaches. In 12 of the 95 cases, the standard retrosigmoid approach was extended to allow additional exposure of the middle fossa through Meckel's cave, which constituted the RISA.

Preoperative, intraoperative, and postoperative records and radiological images obtained in these 12 
patients were reviewed. Data collection included: 1) the patient's neurological status before and after surgery; 2) the radiological features of the tumor and surrounding structures, including size and extension of the tumor, bone changes, and displacement of the brainstem; and 3) the patient's neurological condition at the time of follow-up evaluation. The completeness of tumor resection was assessed intraoperatively and by postoperative imaging and subsequently classified according to the Simpson grading scale of I to V.[24] Each patient's functional status was determined using the Karnofsky Performance Scale at the time of discharge from the hospital. Each patient underwent follow-up medical and radiological examinations. Facial nerve results were graded according to the House--Brackmann grading system.[7]

\section{Surgical Technique}

A suboccipital retrosigmoid craniectomy or craniotomy is performed with the patient in the semisitting position, exposing the edges of the transverse and sigmoid sinuses. Somatosensory evoked potentials, facial electromyographic responses, and brainstem auditory evoked potentials are assessed intraoperatively.

After opening the dura, the cerebellum is slightly elevated and the cerebellomedullary cistern is opened so that CSF can be evacuated. The cerebellum is then held by the retractor medially, and the tumor is exposed in the CPA. Petroclival meningiomas are usually located ventral to the seventh--eighth cranial nerve complex, displacing these nerves downward and backward. Because the tumor may fill the entire CPA and engulf the neurovascular structures, tumor removal is usually performed in the lateromedial direction, starting from the bone and moving toward the brainstem. This approach permits earlier identification of the cranial nerves near their entrance or exit in bone or dura, where their anatomy is somewhat preserved. However, tumor invasion into the foramina may make visualization of cranial nerves difficult. Therefore, persistent piecemeal tumor resection is recommended instead of en bloc tumor removal performed using the cavitron aspirator. Whenever possible, tumor dissection is performed while respecting the arachnoid planes. In a case in which the pia mater of the brainstem is infiltrated by tumor, the policy of complete tumor removal at any price should be avoided.

Drilling the Suprameatal Bone. Once the part of the tumor located in the CPA is removed, resection is performed in the directio of the middle fossa. The fourth cranial nerve is identified and preserved. To enlarge access to Meckel's cave and the middle fossa, the suprameatal portion of the petrous bone is drilled away, over the seventh--eighth cranial nerve complex and dorsolateral to the fifth cranial nerve (Fig. 1). Because of the marked variation in bone anatomy in this region, the amount of bone resection may vary. Meckel's cave is exposed and opened. This allows for mobilization of the trigeminal nerve in the area of the cave both laterally and medially, which increases the surgeon's work space. As many trigeminal nerve fibers as possible are dissected free from the tumor and preserved. If the tumor does not infiltrate the cavernous sinus, complete resection of the portion of tumor located in the middle fossa tumor can be accomplished by dissecting and pulling the tumor downward gradually by using a tumor forceps. In the case of cavernous sinus infiltration, the biological behavior of the tumor will determine the radicality of resection. Meningiomas that compress neurovascular structures can be radically resected, whereas no attempt is made to perform a complete resection of infiltrating growing meningiomas. 


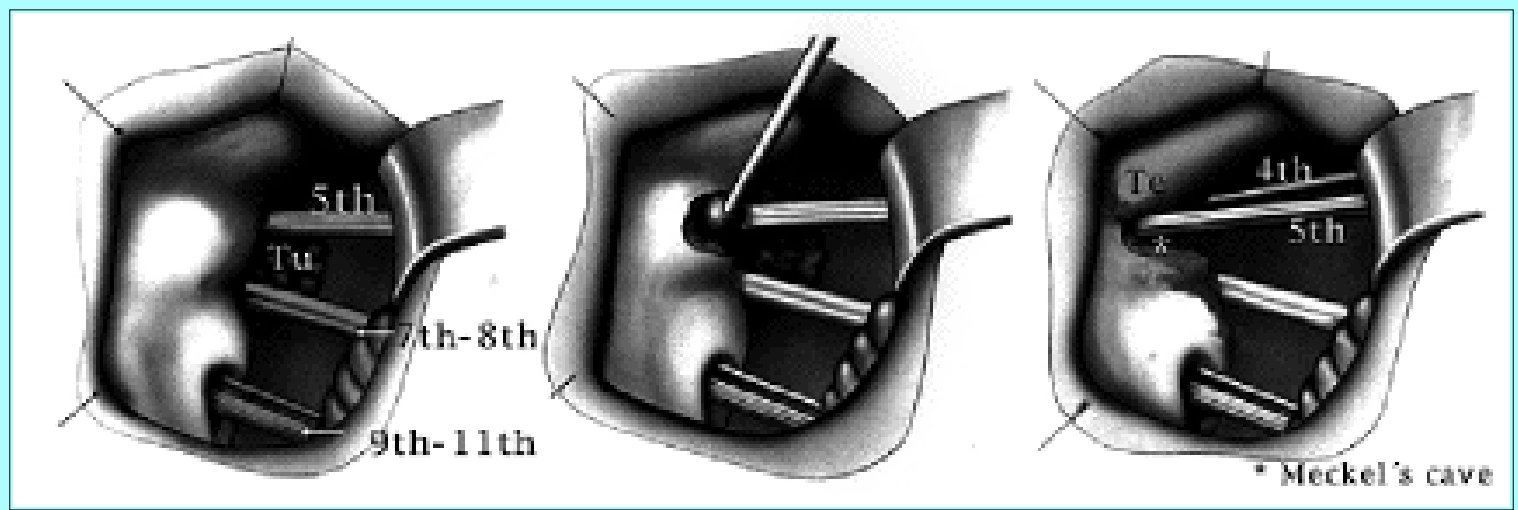

Fig. 1. Schematic drawings showing the procedure by which the suprameatal bone is drilled during the RISA. Surgery is performed with the patient in a semisitting position, and the cerebellum is retracted medially. The nerves running in the CPA are shown. The tumor (Tu) is exposed in the CPA and followed through Meckel's cave, which is opened by drilling the suprameatal bone. Finally, the portion of the tumor within Meckel's cave is resected. If necessary, the tentorium (Te) can be opened above the fifth cranial nerve, which can be mobilized, thus improving access to the middle fossa. Ordinal numbers indicate cranial nerves.

The enlarged approach permits visualization of the oculomotor nerves, the posterior clinoid, the ICAs, the posterior cerebral arteries, and even the optic nerves.

After tumor resection is finished, jugular vein decompression is performed by the anesthesiologist to reveal the presence of any opened veins that might have been unnoticed during surgery performed with the patient in a semisitting position. Once careful homeostasis is accomplished, open bone air cells are sealed with muscle pieces and fibrin glue. The dura is sutured in a watertight fashion, and the craniotomy and wound are closed. Usually, no lumbar drainage is necessary.

\section{ILLUSTRATIVE CASE}

This 50-year-old woman presented with a 2-year history of progressive facial dysesthesia and decreased hearing on the left side.

Examination. Magnetic resonance imaging revealed a homogeneous gadolinium-enhanced mass on the left CPA with slight brainstem compression. The lesion extended through Meckel's cave into the middle fossa (Fig. 2).

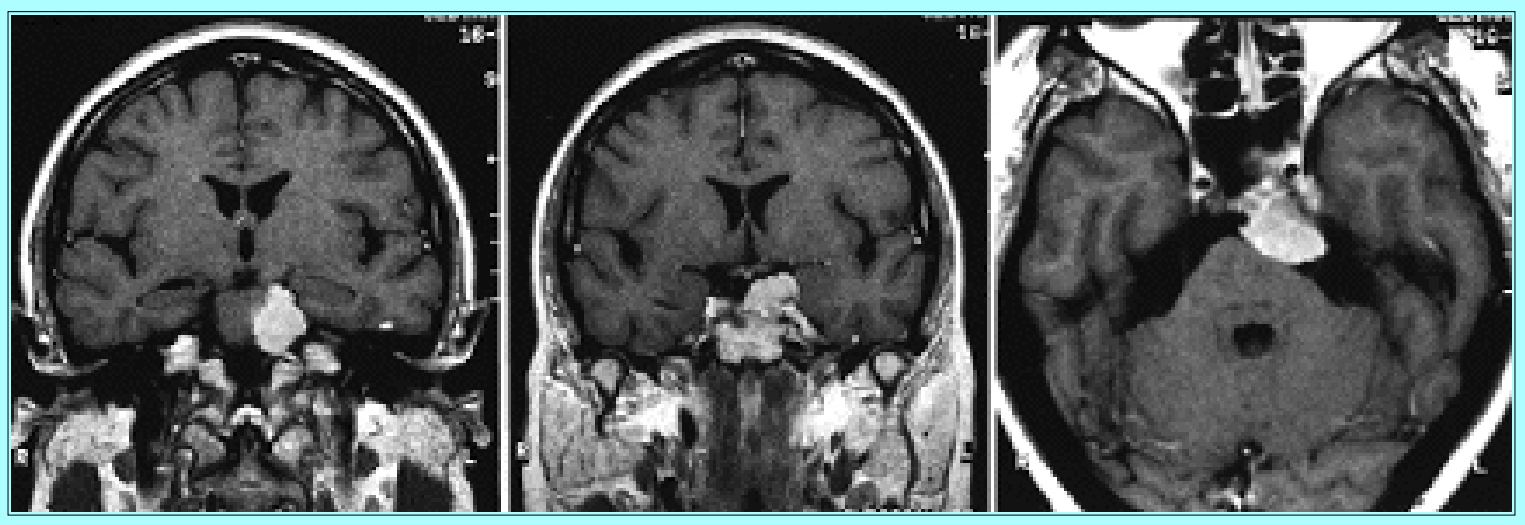

Fig. 2. Preoperative coronal (left and center) and axial (right) contrast-enhanced MR images revealing a homogeneous mass at the left CPA with slight brainstem compression. The 
lesion extends through Meckel's cave into the middle fossa.

Operation. Surgery was performed with the patient in the semisitting position. A retrosigmoid craniotomy was performed, and the CPA was exposed. A vascularized mass was seen between the fifth cranial nerve and the seventh--eighth nerve complex (Fig. 3A and B). Surgical resection was accomplished by drilling the suprameatal bone, opening Meckel's cave, and mobilizing the trigeminal nerve (Fig. 3C--E). Complete resection was achieved with preservation of the surrounding nerves; finally, the drilled bone area was covered with muscle and fixed in place by using fibrin glue (Tissucol; Immuno-Baxter, Heidelberg, Germany) (Fig. 3F and G).

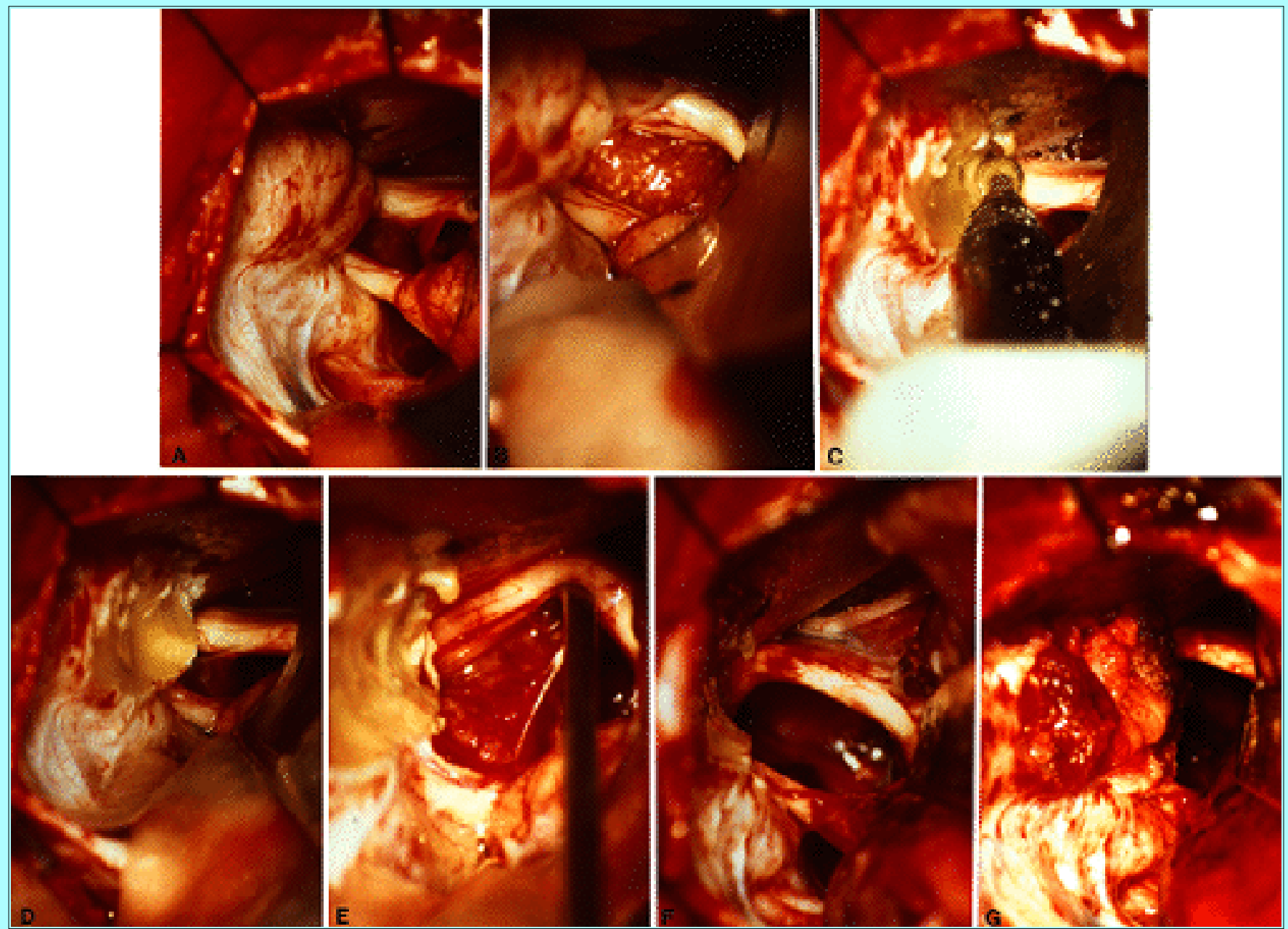

Fig. 3. Intraoperative photographs obtained in the patient represented in Fig. 1, demonstrating tumor exposure and resection via a retrosigmoid craniotomy performed with the patient in a semisitting position. A: View of the left CPA with the fifth, seventh, eighth, ninth, and 10th cranial nerves exposed. The tumor is located between the fifth nerve and the seventh--eighth nerve complex. B: Higher magnification of the view presented in A, showing the tumor between the fifth cranial nerve and the seventh--eighth nerve complex. C: The suprameatal bone is being drilled away. D: Partial resection of the suprameatal bone. E: The trigeminal nerve is mobilized upward to allow additional tumor exposure. F: Complete tumor resection is performed by opening Meckel's cave, and the surrounding nerves are preserved. G: The drilled bone area is covered with muscle and fibrin glue.

Postoperative Course. The patient made an uneventful recovery without additional neurological deficits. Postoperative MR images depicted the results of surgical resection (Fig. 4) and bone-window 
CT scanning results confirmed the drilled bone area at the petrous apex (Fig. 5). Pathological examination revealed a meningotheliomatous meningioma.

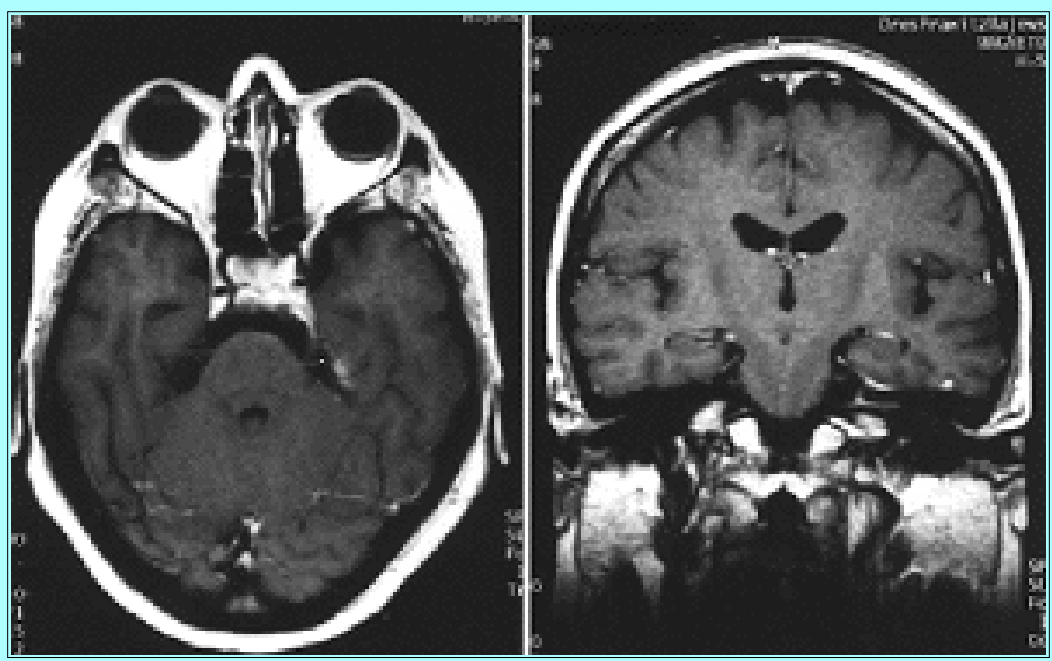

Fig. 4. Postoperative axial (left) and coronal (right) contrast-enhanced MR images confirming complete tumor resection in both the posterior and middle fossae in Meckel's cave.

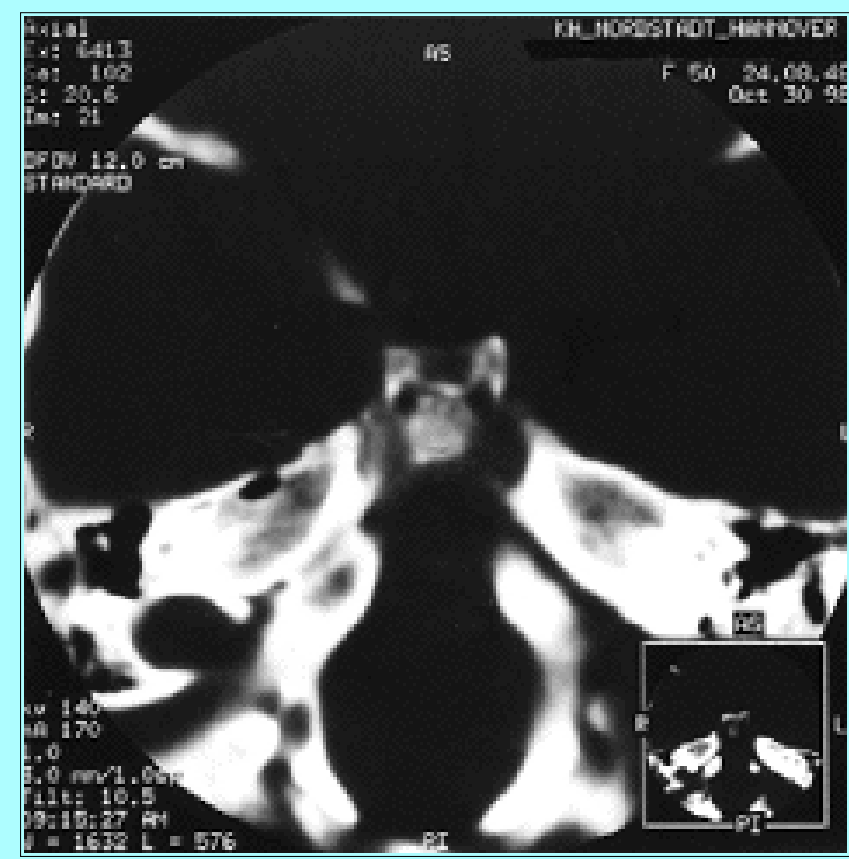

Fig. 5. Postoperative axial CT scan with bone algorithm demonstrating the drilled bone area at the left petrous apex.

\section{RESULTS}

\section{Patient Population}

There were 10 women and two men in this series. Patient ages ranged from 13 to 73 years, with a mean of 48 years. The interval between the onset of symptoms and diagnosis ranged from 1 week to 10 years (mean 1.4 years). The clinical presentations of the patients are shown in Table 1. Hearing loss and dizziness were the most common finding, followed by unsteadiness, tinnitus, and facial hypesthesia. 


\begin{tabular}{|cc|}
\hline \multicolumn{2}{|c|}{ TABLE 1 } \\
OINICAL PRESENTATION OF 12 PATIENTS IN \\
WHOM PETROCLIYL MENINGIOMAS WERE \\
SURGICALLY TREATED VIA THE RISA \\
\hline \hline Symptom orAtnormality & No. of Cas es \\
\hline symptom & \\
hypacusis & 4 \\
dizziness & 4 \\
unsteadiness & 3 \\
innitus & 3 \\
facial hypesthesia & 3 \\
facial pain & 2 \\
headache & 1 \\
di plopia & 1 \\
dysphagia & 1 \\
cranial ner ie atnormality & \\
third & 1 \\
fourth & 1 \\
fifth & 5 \\
sixth & 0 \\
se'venth & 1 \\
eighth & 5 \\
ninth-12th & 1 \\
\hline & \\
\hline
\end{tabular}

\section{Radiological Findings}

In all cases the tumor extended into both the posterior and middle fossae through Meckel's cave and the tentorial notch, affecting the petrous apex and clivus. Nine tumors (75\%) were larger than $30 \mathrm{X} 30 \mathrm{~mm}$, with eight $(67 \%)$ significantly compressing the brainstem. In seven cases the tumor was located ventral to the IAM only, and in five cases the tumor also extended posterior to the IAM. In two patients the tumor had grown into the IAC. In two additional patients the tumor had invaded the cavernous sinus. Bone changes (hyperostosis) were seen in two cases.

Tumor excision measured on MR images had the following diameters: 1) on the axial plane 15 to $50 \mathrm{~mm}$ (mean $25.5 \mathrm{~mm}$ ); on the coronal plane 15 to $32 \mathrm{~mm}$ (mean $24 \mathrm{~mm}$ ); and 3) on the sagittal plane 20 to 34 $\mathrm{mm}$ (mean $26.4 \mathrm{~mm}$ ). The average distance between the brainstem and the posterior surface of the petrous bone at the level of the IAM was $23 \mathrm{~mm}$, as measured using axial contrast-enhanced CT scanning.

\section{Tumor Resection}

The RISA was performed in all 12 cases in this series. Simpson's grades for tumor resection were used to evaluate the completeness of tumor removal. Radical tumor removal (Simpson Grade I or II) was achieved in nine cases (75\%), and subtotal removal (Simpson Grade III) was accomplished in two cases. A patient who presented in a deteriorated general clinical condition harbored a giant infiltrating skull base meningioma that involved the posterior, middle, and anterior fossae; in this particular case (Simpson Grade IV) it was decided to remove only the posterior fossa tumor, which markedly compressed the brainstem.

\section{Postoperative Course}

There were no operative deaths in this series of 12 patients. Postoperative trigeminal hypesthesia 
appeared as a new finding in one patient. Complete facial palsy was observed once immediately after surgery, despite anatomical preservation of the facial nerve; this patient recovered to House--Brackmann Grade 2 within some months. Two additional patients had transient facial weakness, which completely resolved within some weeks. Two patients experienced a decrease in their preoperative hearing levels, but none became deaf after surgery. All but one patient were completely independent at the time of discharge from the hospital (Karnofsky Performance Scale Score 80--90).

All patients received postoperative follow-up evaluations, which ranged from 6 months to 9 years (mean 5.6 years; median 2.7 years). All patients but one are following their normal life activities without difficulties. The one exception is a patient who had been dependent on nursing assistance before surgery due to his deteriorated clinical condition. This patient, who had harbored a giant skull base meningioma, became independent within the 1st postoperative year, although he still experienced some difficulties. None of the patients who underwent radical tumor resection has experienced tumor recurrence to date. Tumor regrowth was observed 8.5 years after surgery in one patient who underwent a Simpson Grade III resection. Because this patient has only minor symptoms, the tumor is under observation.

\section{DISCUSSION}

The petroclival region and Meckel's cave have become more accessible since recent developments in skull base approaches that involve variable resection of the petrous bone, some of which include combined approaches. In 1973 Morrison and King[12] described the combined transtentoral approach by using a translabyrinthine petrosectomy. Later reports contain descriptions of modern combined transpetrosal approaches with preservation of the sigmoid sinus.[2,13,14] The advantages of the combined approaches include a wide surgical field, multiple axes of dissection, minimal brain retraction, and early access to feeding vessels. However, major disadvantages are increased risk of damage to inner ear structures and the facial nerve, temporal lobe retraction, increased risk of injury to the vein of Labbé, and increased operative time.

Aware of these problems, the senior author has modified the classic retrosigmoid approach to access the compartment of the middle fossa via the infratentorial space by drilling the portion of the petrous bone that is located anterior to and above the IAM. This approach is called RISA, and it was used at our instituion for the first time in 1983.

In 1995, Cheung and coworkers[5] reported an approach similar to the RISA. They described the surgical technique of a retrosigmoid craniotomy followed by drilling between the seventh--eighth nerve complex and the fifth cranial nerve. Their technique is illustrated in a report of a case of trigeminal schwannoma.[5]

The bone area that is resected using the RISA is, in a particular sense, similar to the area exposed extradurally through the middle fossa by using the so-called "Kawase approach."[9] In 1985 Kawase and colleagues[9] described the anterior subtemporal transpetrosal--transtentorial approach with selective epidural resection of the anterior pyramid ventral to the IAC. Later, in 1991, these authors[8] published a report on a series of 10 patients in whom petroclival meningiomas were resected using this approach. The area of bone resection is limited by the trigeminal impression anteriorly, the ICA and greater superficial petrosal nerve laterally, and the IAC posteriorly. Using the RISA the direction from which the bone area is drilled is posterior and below, whereas using Kawase's approach it is anterior and above.

Dumbbell-shaped trigeminal schwannomas usually expand the bone surrounding Meckel's cave, creating 
a natural enlarged space between the posterior and middle fossae. This is the space that is accessed using the RISA, by drilling away the suprameatal bone dorsocaudal and lateral to Meckel's cave. Recently, Seoane and Rhoton[23] pubished a nice description of the microsurgical anatomy of the suprameatal approach, detailing important neurovascular structures and landmarks related to this approach.

The RISA permits removal of some petroclival tumors, located mainly in the posterior fossa, that extend through Meckel's cave into the middle fossa, reaching structures such as the third cranial nerve, the ICA, and the optic nerves. Major advantages of using the RISA include avoidance of wide supratentorial craniotomy and temporal bone retraction. $[5,19]$ The retrosigmoid route of the RISA provides early visualization of cranial nerves, principally the lower cranial nerves. The presence of large tumors increases the working space within the CPA. Because the majority of these tumors displace the seventh--eighth cranial nerve complex downward and, in cases of meningiomas with a matrix at the petrous apex, the trigeminal nerve upward, the usually narrow anatomical space[10] is greatly enlarged, which facilitates tumor resection. In the majority of cases the petrosal vein is markedly displaced and/or compressed by tumor and collateral veins have developed, so that the petrosal vein usually does not represent an obstacle for the RISA procedure. It can be coagulated and transected.

An additional advantage of the RISA is the possibility of mobilizing the trigeminal nerve after opening the bone space to Meckel's cave. Mobilization of the trigeminal nerve improves the chances of preserving it while achieving total tumor resection. In addition, the RISA provides an earlier identification of the sixth cranial nerve at the brainstem during tumor dissection, in comparison with the lateral transpetrosal approaches.

\section{Patient Selection}

Presently, we use the following criteria to select which patients harboring petroclival meningiomas should undergo surgery via the RISA: 1) patients with large posterior fossa tumors that have minor extension into the middle fossa; 2) patients with tumors that extend into both the posterior and middle fossae, but exhibit no radiological sign of invasion into the cavernous sinus; 3 ) patients with tumors that extend into both the posterior and middle fossae and invade the cavernous sinus, in whom surgery of the cavernous sinus is not attempted; and 4) geriatric patients in a deteriorated clinical condition, in whom brainstem decompression becomes the major goal of the surgery.

The RISA is especially indicated to remove soft tumors in which the arachnoid planes are preserved, such as some meningiomas and trigeminal schwannomas with extensive posterior fossa extension. Therefore, careful patient selection is mandatory so as not to extend the use of RISA beyond its real possibilities.

Based on these selection criteria, the extent of surgical excision achieved using the RISA in cases of petroclival meningiomas was comparable to that achieved using more extensive, transpetrosal approaches.[1,3,17,22,25] The major surgical complications related to transpetrosal approaches[26] could be avoided in this series by using the RISA. There was no incidence of operative mortality, and the rate of postoperative morbidity was relatively low in this series.

\section{CONCLUSIONS}

The modification of the retrosigmoid approach presented here seems to represent a development toward less invasive surgical treatment of a special group of petroclival tumors. We believe that with careful selection of cases, extensive transpetrosal procedures can be avoided in a number of patients and can be 
replaced by the RISA with very acceptable postoperative results.

\section{References}

1. Al-Mefty O, Ayoubi S, Smith RR: The petrosal approach: indications, technique, and results. Acta Neurochir Suppl 53:166-170, 1991

2. Al-Mefty O, Fox JL, Smith RR: Petrosal approach for petroclival meningiomas. Neurosurgery 22:510-517, 1988

3. Bricolo AP, Turazzi S, Talacchi A, et al: Microsurgical removal of petroclival meningiomas: a report of 33 patients. Neurosurgery 31:813-828, 1992

4. Cantore G, Delfini R, Ciappetta P: Surgical treatment of petroclival meningiomas: experience with 16 cases. Surg Neurol 42:105-111, 1994

5. Cheung SW, Jackler RK, Pitts LH, et al: Interconnecting the posterior and middle cranial fossae for tumors that traverse Meckel's cave. Am J Otol 16:200-208, 1995

6. Hakuba A: The transpetrosal-transtentorial approach and its application in the therapy of retrochiasmatic craniopharyngiomas, in Samii M (ed): Surgery in and Around the Brain Stem and the Third Ventricle. Anatomy, Pathology, Neurophysiology, Diagnosis, Treatment. Berlin: Springer-Verlag, 1986, pp 396-402

7. House JW, Brackmann DE: Facial nerve grading system. Otolaryngol Head Neck Surg 93:146-147, 1985

8. Kawase T, Shiobara R, Toya S: Anterior transpetrosal-transtentorial approach for sphenopetroclival meningiomas: surgical method and results in 10 patients. Neurology 28:869-876, 1991

9. Kawase T, Toya S, Shiobara R, et al: Transpetrosal approach for aneurysms of the lower basilar artery. J Neurosurg 63:857-861, 1985

10. Lang J: Clinical Anatomy of the Posterior Cranial Fossa and its Foramina. Stuttgart: Thieme, 1991

11. Miller CG, van Loveren H, Keller JT, et al: Transpetrosal approach: surgical anatomy and technique. Neurosurgery 33:461-469, 1993

12. Morrison AW, King TT: Experiences with a translabyrinthine-transtentorial approach to the cerebellopontine angle. Technical note. J Neurosurg 38:382-390, 1973

13. Samii M, Ammirati M: The combined supra-infratentorial pre-sigmoid sinus avenue to the petro-clival region. Surgical technique and clinical applications. Acta Neurochir 95:6-12, 1988

14. Samii M, Ammirati M, Mahran A, et al: Surgery of petroclival meningiomas: report of 24 cases. Neurosurgery 24:12-17, 1989

15. Samii M, Carvalho GA, Tatagiba M, et al: Surgical management of meningiomas originating in Meckel's cave. Neurosurgery 41:767-775, 1997 
16. Samii M, Migliori MM, Tatagiba M, et al: Surgical treatment of trigeminal schwannomas. J Neurosurg 82:711-718, 1995

17. Samii M, Tatagiba M: Experience with 36 surgical cases of petroclival meningiomas. Acta Neurochir 118:27-32, 1992

18. Samii M, Tatagiba M: Neurosurgical aspects of tumors of the base of the skull, in Youmans JR (ed): Neurological Surgery, ed 4. Philadelphia: WB Saunders, 1996, pp 3024-3040

19. Samii M, Tatagiba M, Carvalho GA: Resection of large petroclival meningiomas by the simple retrosigmoid route. J Neurosurg Sci 6:27-30, 1999

20. Samii M, Tatagiba M, Piquer J, et al: Surgical treatment of epidermoid cysts of the cerebellopontine angle. J Neurosurg 84:14-19, 1996

21. Sanna M, Mazzoni A, Saleh EA, et al: Lateral approaches to the median skull base through the petrous bone: the system of the modified transcochlear approach. J Laryngol Otol 108: 1036-1044, 1994 22. Sekhar LN, Pomeranz S, Sen CN: Extradural petrous bone and petroclival neoplasms. Acta Neurochir Suppl 53:183-192, 1991

23. Seoane E, Rhoton AL Jr: Suprameatal extension of the retrosigmoid approach: microsurgical anatomy. Neurosurgery 44:553-560, 1999

24. Simpson D: The recurrence of intracranial meningiomas after surgical treatment. J Neurol Neurosurg Psychiatry 20:22-39, 1957

25. Spetzler RF, Hamilton MG, Daspit CP: Petroclival lesions. Clin Neurosurg 41:62-82, 1994

26. Tatagiba M, Samii M, Matthies C, et al: Management of petroclival meningiomas: a critical analysis of surgical treatment. Acta Neurochir Suppl 65:92-94, 1996

27. Tedeschi H, Rhoton AL Jr: Lateral approaches to the petroclival region. Surg Neurol 41:180-216, 1994

Manuscript received April 21, 1999.

Accepted in final form October 12, 1999.

Address reprint requests to: Madjid Samii, M.D., Neurochirurgische Kliniken, Medizinische Hochschule Hannover und Klinikum Hannover Nordstadt, Carl-Neuberg-Strasse 1, D-30625 Hannover, Germany. email: neurosurgery.nordstadt@metronet.de. 